\title{
Sentido de número na infância: uma interconexão dinâmica entre conceitos e procedimentos
}

\author{
Heloiza Helena de Jesus Barbosa \\ Universidade Federal de Santa Catarina, Florianópolis-SC, Brasil
}

Resumo: A presente revisão tem o objetivo de analisar o que estudos acadêmicos e experimentais têm revelado sobre os processos cognitivos de desenvolvimento envolvidos na conceituação de número pela criança. Presentemente, dois grupos de orientações teóricas opostas têm argumentado sobre a natureza da habilidade numérica das crianças. Apesar de inconcluso o debate, estudos revistos neste artigo parecem apontar para a existência de uma interconexão entre conceitos e procedimentos no processo de construção do sentido de número. Veremos, também, como a qualidade do sentido de número construído pelas crianças pode estar relacionada com o desempenho acadêmico na área da matemática.

Palavras-chave: Sentido de número. Desenvolvimento numérico. Desenvolvimento cognitivo. Educação infantil.

\section{Sense of numbers in early childhood: a dynamic interconnection between concepts and procedures}

\begin{abstract}
The present review describes what academic and experimental studies in mathematics have revealed about cognitive processes in young children's numerical abilities. Currently, two groups with opposite theoretical approaches are discussing the nature of children's numerical abilities. Although the debate is far from being conclusive, the studies revised in this article seem to indicate that there is a dynamic interconnection between concepts and procedures in the process of constructing the sense of number. In addition, it is discussed how the quality of the number sense constructed by the child might be connected with his/her school math achievement.
\end{abstract}

Keywords: Number sense. Numerical development. Cognitive development. Early childhood education.

\section{El sentido del número en la infancia: una interconexión dinámica de conceptos y procedimientos}

Resumen: La revisión presentada se ocupa de analizar qué nos revelan los estudios académicos y experimentales sobre procesos cognitivos de desarrollo implicados en la conceptuación de número hecha por los niños. Presentemente, dos grupos de orientaciones teóricas opuestas han argumentado sobre la naturaleza de la habilidad numérica de los niños. Aunque el debate esté todavía abierto, investigaciones revistas en este artículo parecen apuntar para la existencia de una interconexión entre conceptos y procedimientos en el proceso de construcción de sentido de número. Veremos, también, como la calidad del sentido de número construido por niños puede estar relacionada con su desempeño académico en matemática.

Palabras clave: Sentido de número. Desarrollo numérico. Desarrollo cognitivo. Educación infantil. 
Recentemente, tem-se discutido muito sobre o sentido de número como um novo paradigma de pesquisa e ensino no campo da matemática. Este termo traduzido do inglês number sense e que tornouse popular no meados dos anos 90, apresenta variadas significações no campo da psicologia cognitiva e do desenvolvimento, como também, no campo dos estudos sobre ensino e aprendizagem que precisam ser elucidadas.

Dentro dos estudos psicológicos sobre o desenvolvimento do senso numérico há controversias sobre a natureza desta sensibilidade quantitativa. Enquanto um grupo de pesquisadores argumenta sobre a base inatista da especificidade do módulo numérico (Butterworth, 1999; Dehaene, 1997), um segundo grupo defende o desenvolvimento do sentido de número com base em habilidades cognitivas genéricas (Fuson, 1988; Mix \& Sandhofer, 2007). Ambos os grupos apresentam dados empíricos de estudos com bebês que serão revistos no decorrer deste artigo. Como também, serão analisadas as implicações de uma ou outra posição nas viviências numéricas dentro da educação infantil.

No que se refere ao segundo campo mencionado, o termo sentido de número relaciona-se a apreensão contextualizada de conceitos e procedimentos lógicos-matemáticos envolvendo números e quantidades, mesmo antes da formalização do ensino da matemática pela escola. Pesquisas sobre o desenvolvimento do sentido de número têm revelado que existe uma relação estreita entre o desenvolvimento desta conceituação numérica e o desempenho acadêmico na área da matemática (Nunes \& Bryant, 1997; Spinillo, 2006). Estes estudos também serão revistos no decorrer do presente artigo.

Este artigo pretende contribuir para o debate acerca dos processos cognitivos de formação do conceito de número, como também, tornar acessível ao professor e/ou ao profissional da educação infantil informações coletadas por estudos acadêmicos que dizem respeito ao desenvolvimento de conceitos e procedimento numéricos na criança. Acredita-se que estas informações podem auxiliar o professor em sua atuação pedagógica. Para atingir tal objetivo, serão apresentados resultados de estudos que investigaram o desenvolvimento numérico infantil, e uma análise crítica dos mesmos. A trajetória desses estudos e os debates que os mesmos suscitaram, como também, os possíveis caminhos para futuras investigações, serão abordados.

\section{Representações quantitativas não-verbais - são estas numéricas?}

Tem sido amplamente divulgado pela mídia que os bebês humanos têm habilidades matemáticas surpreendentes como cálculo, contagem e comparação. Mas, o que estes relatos jornalísticos não publicaram foi uma análise crítica dos estudos que demonstraram tais habilidades. São os bebês humanos realmente capazes de reconhecer diferentes magnitudes? Os bebês sabem que o numeral 2 é mais que 1 , e que 2 é menos que 3? Pesquisadores investigando o desenvolvimento cognitivo infantil ponderaram essas mesmas perguntas e estudos sobre o desenvolvimento numérico em bebês foram conduzidos. Revisaremos estes estudos em seguida.

Para estudar o desenvolvimento quantitativonumérico em bebês, pesquisadores inicialmente tiveram que lidar com dificuldades metodológicas. A metodologia usada com crianças mais velhas, que já sabem falar, é inadequada para se estudar bebês. Como conseguir obter e documentar respostas de um bebê que ainda não fala?

Pesquisadores responderam a este desafio criando uma metodologia de estudo que é baseada no comportamento mais proeminente dos bebês, que é o de olhar as coisas ao redor. Essa nova metodologia foi chamada de habituação (KarmiloffSmith, 1992). A metodologia da habituação é baseada na premissa de que bebês procuram e se deleitam em encontrar novidades. Esta busca guia o olhar do bebê. É assumido, então, que bebês olham mais intensamente para as coisas que são novas do que para as coisas as quais eles já estão habituados. A metodologia da habituação/desabituação funciona da seguinte forma: é apresentado ao bebê um estímulo que se repete por várias vezes até o mesmo mostrar desinteresse e passar a olhar por curto tempo para o referido estímulo. Neste momento, um novo estímulo é mostrado ao bebê. Se o bebê mostrar interesse por esse novo estímulo, olhando-o por um tempo mais longo, então, pode ser concluído que o novo estímulo 
foi apreendido (percebido, entendido) pelo bebê como diferente do estímulo inicial. A duração do olhar do bebê ao novo estímulo é, então, medida para verificar se o mesmo demonstra ou não interesse no novo estímulo.

Pesquisadores, manipulam diversas variáveis para, desta forma, determinarem qual é a variável que realmente desperta o interesse do bebê e reengaja a atenção do mesmo. No caso de pesquisas sobre o desenvolvimento numérico, os pesquisadores habituam e desabituam os bebês às variáveis numéricas para argumentar que nos seus experimentos os bebês prestaram atenção ao aspecto numérico.

$\mathrm{O}$ primeiro estudo, com o objetivo de investigar a habilidade dos bebês para discriminar numerosidades, foi conduzido por Starkey e Cooper (1980). Neste estudo, os bebês de 5 a 10 meses de idade permaneciam sentados no colo de suas mães e eram habituados a slides mostrando 1 bola preta (grupo 1) ou 3 bolas pretas (grupo 2) organizadas em alinhamento horizontal. Quando os bebês começavam a olhar para todas as outras coisas ao redor, menos para o slide, pois eles já estavam desinteressados, a fase da habituação terminava e começava então a fase do teste. $\mathrm{Na}$ fase do teste, os pesquisadores primeiro mostravam novos slides com a mesma quantidade de bolas pretas da fase inicial de habituação, mas manipulavam o espaço entre as mesmas (mais e menos espaço entre as bolas). Quando os bebês começaram a mostrar desinteresse por esses slides, os slides mudavam abruptamente para números de bolas pretas diferentes dos mostrados na fase inicial de habituação. $\mathrm{O}$ tempo de duração do olhar foi medido, e os resultados dessas medidas demonstraram que os bebês olhavam por mais tempo para a mudança numérica do que para a mudança espacial. Entretanto, não foram todas as mudanças numéricas que foram percebidas pelos bebês. Os bebês deste estudo não demonstraram perceber as mudanças numéricas entre quatro e seis itens. Starkey e Cooper (1980), então, concluíram que antes dos dez meses de idades os bebês são capazes de discriminar quantidades pequenas (entre 1 e 2 , e 2 e 3). Como os bebês fazem isso? Os pesquisadores sugeriram que bebês humanos usam o processo de subitizing para discriminar quantidades pequenas. Subitizing é um processo de discriminação visual em que é possível detectar até três objetos com uma rapidez de milésimos de segundos (Chi \& Klahr, 1975), a qual não requer uma contagem consciente.

Este primeiro estudo inspirou muitos outros experimentos que seguiram a mesma metodologia. Por exemplo, Strauss e Curtis (1981) usaram a mesma metodologia e os procedimentos básicos de Starkey e Cooper (1980) para testar bebês de cinco a doze meses de idade. Mas, para poderem controlar outras variáveis perceptuais, eles usaram fotos de objetos com variação de tamanho, cor, forma, perspectiva, localização no espaço, e número de objetos (quantidade). Eles detectaram que dentre todas essas variáveis, somente a variável número de objetos (dois e três objetos) causou o aumento da duração do olhar do bebê para a tela de projeção.

Se os bebês apresentam essa competência aos cinco meses de idade, então, eles também deveriam apresentar a mesma competência alguns dias após o nascimento. Este foi o argumento usado por Antell e Keating (1983) para conduzir o experimento no qual eles apresentavam a crianças, com poucos dias de nascidas, dois cartões com bolas pretas impressas. As bolas eram do mesmo tamanho, mas em um cartão as bolas estavam arrumadas horizontalmente e com um grande espaço entre elas; no outro cartão, as bolas estavam bem próximas umas das outras. Depois de ter habituado os recém-nascidos a este estímulo, foilhes apresentado um terceiro cartão que mantinha a disposição espacial constante, mas mudava o número de bolas de duas para três. Os recém-nascidos reengajaram a atenção em resposta à variação numérica em vez da variação espacial.

Mas será que foi o aspecto numérico que reengajou a atenção dos bebês e possibilitou a discriminação de quantitades? Com o intuito de responder a esta pergunta, os estudos com bebês não pararam aí. Vários outros experimentos foram feitos, nestes quase trinta anos desde a publicação do primeiro estudo. Diferentes grupos se estabeleceram defendendo posições também diferentes quanto a natureza do sentido de número presente no bebê.

Por exemplo, um grupo de pesquisadores argumenta que o quê os bebês notam nos estudos de habituação são alterações numéricas baseadas no conhecimento conceitual de número de natureza inata 
(Carey, 1991; Carey, 2001; Carey \& Spelke, 1994; Gelman, 1991; Gelman \& Gallistel, 1978; Gelman \& Meck, 1983; Spelke, Breinlinger, Macomber \& Jacobson, 1992; Spelke \& Tsivkin 2001; Starkey \& Spelke, 1983; Wynn, 1992b, 1992c, 1998a, 1998b). Estes pesquisadores fazem parte da linha teórica do nativismo, ou inatismo. A abordagem inatista afirma que o desenvolvimento de conceitos numéricos envolve o movimento de acesso a conhecimentos matemáticos inatos localizados em módulo cerebral específico para números (Butterworth, 1999; Dehaene, 1997; Spelke \& Dehaene, 1999). Assim, para pesquisadores desta abordagem, os bebês prestam atenção em informações numéricas ao redor deles porque seus cérebros são equipados desde o nascimento para fazê-lo.

Por outro lado, um diferente grupo de pesquisadores diz que o quê talvez os pesquisadores nativistas chamem de "conhecimento numérico inato" nada mais são do que processos de domínios gerais que auxiliam o desenvolvimento de várias atividades cognitivas, sem serem exclusivos a um único domínio como, por exemplo, número (Clearfield \& Mix, 1999, 2001; Cooper, 1984; Huttenlocher, Duffy \& Levine, 2002; Mix, Levine \& Huttenlocher, 1997; Mix, Sandhofer \& Baroody, 2005; Strauss \& Curtis, 1984; Wakeley, Rivera \& Langer, 2000). De acordo com esta perspectiva, a atividade perceptiva do bebê que auxilia nos processos de individuação do objeto, adicionada à atividade de aquisição dos nomes dos numerais que auxilia na centração da atenção nos aspectos numéricos, podem levar à formação de categorias conceituais de número sem ser necessário a existência de um módulo numérico específico, de natureza inata.

Estudos produzidos por este grupo de pesquisadores, contrários às idéias nativistas, mostram que nenhum estudo foi capaz de desvincular as dimensões perceptuais do mundo físico dos aspectos numéricos conceituais (Clearfield \& Mix, 1999, 2001; Wakeley, Rivera \& Langer, 2000). Estes pesquisadores afirmam que a metodologia de habituação não demonstra, indubitavelmente, que o comportamento do bebê de olhar mais intensamente para algo reflete um conhecimento pré-existente deste algo. Pois, o próprio ato de habituar pode tornar relevante aspectos ou variáveis que antes não o eram (Strauss \& Curtis, 1984). Ou seja, expor o bebê a um estímulo que se repete (chegando a trinta vezes) pode criar uma atenção ao aspecto numérico que não existia antes. Portanto, isso não quer dizer que o bebê tenha a capacidade inata de perceber número, mas talvez o aspecto numérico passou a ser relevante dentro do próprio contexto do experimento que isola e enfatiza o aspecto numérico. Cooper (1984) pondera que os resultados dos estudos com bebês não sustentam a afirmação de que um bebê "sabe" o que 1, 2 ou 3 bolas significam numericamente. Os bebês podem perceber que um conjunto de dois objetos é diferente de um conjunto com três objetos, mas isso não quer dizer que eles saibam exatamente o que as quantidades "dois" e "três" significam. Clearfield e Mix (1999) confirmaram essa ponderação de Cooper (1984) em um experimento onde a densidade visual do estímulo foi separada da variável de quantidade numérica. Os resultados mostraram que bebês são sensíveis às mudanças de densidade, comprimento, e ocupação espacial (perceptivas) e não às mudanças numéricas. Replicações do estudo de Clearfield e Mix (1999) obtiveram os mesmos resultados (Feigenson, Carey \& Spelke, 2002).

Assim, a explicação inatista de que conhecimento numérico inato guia a performance dos bebês em atividades de discriminação de quantidades perde sua força com as recentes evidências que demonstraram que os bebês, na verdade, usam informações perceptuais sobre objetos e a ocupação dos mesmos no espaço para elaborar representações de individuação e agrupamento dos mesmos (Mix \& Sandhofer, 2007). Desta forma, as representações mentais criadas pelos bebês humanos que envolvem quantidade não são, necessariamente, guiadas por conhecimento ou conceito numérico inato, mas podem ser representações gerais advindas das habilidades perceptivas gerais e que podem posteriormente influenciar o desenvolvimento matemático (Mix, Huttenlocher \& Levine, 2002a, 2002b; Mix \& Sandhofer, 2007).

Apesar das controvérsias, os estudos com bebês demonstraram, indubitavelmente, que desde cedo os bebês estão atentos para as informações de densidade, luz, brilho, volume, quantidade e som, por 
exemplo. Este conhecimento quantitativo pré-verbal deve ser levado em consideração por profissionais da educação infantil na hora de planejar ações educativas para as crianças mais novas de creche e/ ou pré-escola. Ou seja, não é porque a criança ainda não sabe contar verbalmente que ela não está construindo conceitos quantitativos ao interagir com objetos do mundo físico. Os estudos mostraram exatamente o contrário. Levar os resultados dos estudos em consideração, então, significa planejar e mediar situações de aprendizagem nas quais os aspectos quantitativos de natureza pré-verbal (antes da contagem oral) possam ser experienciados, evidenciados e registrados por todos os participantes de forma lúdica, simbólica e prazerosa.

\section{$O$ ato de contar}

Das representações quantitativas iniciais para a contagem verbal ocorre um longo e complexo processo de desenvolvimento. A aquisição dos nomes dos numerais, como também, a aquisição dos procedimentos de contagem e o entendimento de porque e o quê contar, requer a junção de vários conhecimentos por parte do sujeito cognoscente, de ordens conceitual e prática. Vejamos o que pesquisadores descobriram até então sobre este complexo processo de desenvolvimento.

Quem já passou algum tempo observando uma criança de dois anos brincando, pôde notar que quando as crianças verbalizam numerais em suas brincadeiras estes não necessariamente correspondem à exata quantificação ou, até mesmo, à correspondência um-para-um. Muitas vezes, a verbalização dos numerais nesta idade reflete a experimentação do uso de novas palavras sem o estabelecimento, ainda, da conexão conceitual numeral-quantidade. Por exemplo, uma certa vez Francisco de dois anos e meio de idade, filho da autora deste artigo, estava arrumando um grupo de animais dentro do castelo feitos de blocos de papelão imitando tijolos. Ao terminar de colocar todos os animais dentro do castelo (aproximadamente doze animais), a criança anunciou "Tem três animais dentro do castelo!" A mãe de Francisco, que acompanhava a brincadeira, interveio: "Eu acho que tem mais que três animais dentro do castelo. Você pode contar para ver se tem três?" A criança passou os dedos rapidamente em volta de todos os animais e recitou as seguintes palavras sem pausa entre elas "um-doistrês-quatro-cinco-seis-sete-oito-nove-dez." A mãe então perguntou: "Quantos animais têm?" Francisco respondeu: "Três!"

A observação deste comportamento comum em crianças pequenas levou vários pesquisadores a estudar a contagem e a relação desta com o desenvolvimento de conceitos de número.

Jean Piaget e a pesquisadora Alina Szeminska ${ }^{1}$ (Piaget \& Szeminska, 1975) foram os primeiros a estudar, através do método clínico, a complexidade envolvida na formação do conceito de número. Entretanto, inicialmente, não atribuíram nenhuma função cognitiva importante para esta recitação verbal dos numerais, pois eles estavam preocupados em documentar os processos lógicos de conceituação numérica que, segundo eles, seriam a base para construção dinâmica e constante da inteligência humana.

Os estudos pós-piagetianos, principalmente os estudos feitos nos Estados Unidos, são marcados pelo debate em torno de duas questões: 1) do papel da contagem na construção do sentido de número e, 2) da natureza do conceito de número; ou seja, se este conceito é de natureza conceitual inata ou é um procedimentos adquirido.

Os vários estudos conduzidos tendo em vista responder as duas questões mencionadas acima acentuaram diferenças teóricas e epistemológicas entre os investigadores de orientação inatista e os investigadores que enfatizam os processos de desenvolvimento. Veremos em seguida como esses dois grupos explicam as mudanças que ocorrem na passagem das habilidades quantitativas pré-linguísticas dos bebês para as habilidades numéricas mediadas pela linguagem oral das crianças.

Os pesquisadores americanos Gelman e Gallistel (1978), utilizando-se de observações e testes laboratoriais, perceberam que as ações de contar das crianças não são aleatórias mas, ao contrário,

\footnotetext{
${ }^{1}$ A pesquisadora Alina Szeminska não teve seu nome incluído na tradução do livro sobre o desenvolvimento dos conceitos numéricos em inglês.
} 
apresentam uma organização. Estes investigadores sugeriram que o ato de contar, mesmo sendo restrito inicialmente a pequenas quantidades e com erros na seqüência de ordenação numeral, é guiado por conhecimento inato dos princípios da contagem. Esses princípios são:

1) Princípio da correspondência de um-paraum: crianças sabem que cada objeto pode ser contado somente uma vez.

2) Princípio da ordem estável: os numerais devem ser usados em uma ordem fixa e estável.

3) Princípio da cardinalidade: o último numeral falado significa a quantidade de objetos presentes no conjunto contado.

4) Princípio da irrelevância da ordem de contagem: os objetos podem ser contados da direita para esquerda, da esquerda para a direita, de cima para baixo, de baixo para cima, do meio para a esquerda, ou do meio para direita; enfim, de qualquer jeito sem que isso altere o resultado da contagem.

5) Princípio da generalização: os mesmos princípios são usados para contar qualquer conjunto, seja este composto de objetos, ações ou sons.

Apesar de os estudos de Gelman e Gallistel (1978), por um lado, terem mostrado que o ato de contar envolve ações cognitivas complexas, por outro lado, suas conclusões de que estes conhecimentos estão presentes na mente da criança desde o nascimento parecem extremas e sem apoio empírico.

Uma outra pesquisadora, Fuson (1988), publicou um livro que reuniu os resultados de estudos feitos no seu laboratório com a colaboração de outros pesquisadores durante os anos de 1980 a 1988 (Fuson, Richards \& Briars, 1982; Fuson, Secada \& Hall, 1983; Fuson, Pergament, Lyons \& Hall, 1985). Seus resultados mostraram que crianças de três a cinco anos de idade, idade bem menor do que as crianças estudadas por Gelman e Gallistel (1978), são capazes de contar corretamente um conjunto com pequena quantidade de itens sem saber que o último numeral falado representa a quantidade do conjunto (cardinalidade). Isto acontece desta forma: as crianças contam um conjunto com três itens; quando perguntadas sobre quantos itens há no conjunto que acabaram de contar, elas, geralmente, ou re-contam os itens ou dizem um numeral que não foi o último numeral falado por elas, ou ainda dizem uma outra seqüência de numerais. Assim, as crianças pesquisadas por Fuson (1988) parecem não ter o princípio de cardinalidade inato como sugeriram Gelman e Gallistel (1978). Esses dados de pesquisa colocam em dúvida os argumentos nativistas.

Em Fuson (1988) e em Fuson e cols. (1982) também é mostrado que a seqüência de numerais recitados durante a contagem passa por modificações durante o processo de desenvolvimento. Por exemplo, uma criança pode recitar a seguinte seqüência "umdoistrêsquatrocincoseis" em que as palavras não são diferenciadas. Posteriormente, ela começa a diferenciar as palavras "um - dois - três - quatro cinco - seis" Em seguida, a criança começa a associar a palavra dita ao objeto referente em uma relação de um-para-um sem a produção de um resultado cardinal. Como também, a criança pode produzir a seguinte seqüência "um, dois, três, cinco, quatro, sete" na qual os três numerais iniciais são parte de uma seqüência convencional e os três últimos de uma seqüência não-convencional com omissão. Com a aquisição de mais palavras para designar numerais, essa seqüência se modifica e pode tornar-se mais convencional, mas ainda sem o entendimento da função da contagem (i.e., estabelecer quantidades). Se essa transformação ocorre na produção da seqüência numérica desacompanhada do entendimento conceitual, então este conhecimento não pode ser considerado inato, ou mesmo guiado por conceitos inatos, como sugeriram Gelman e Galistell (1978).

Além de demonstrar problemas com o argumento inatista para explicar os princípios da cardinalidade e da ordem estável, pesquisas de Fuson e seus colegas (Fuson e cols., 1983, 1985) também mostraram que as crianças pequenas cometem erros durante a contagem, violando assim o princípio de correspondência um-para-um. Os erros mais comuns são: 1) apontar para o objeto sem dizer o numeral correspondente, ou dizer o numeral sem apontar; 2) apontar para um objeto mais de uma vez, ou deixar um objeto sem apontar; 3) apontar para um objeto duas vezes e dar-lhe três palavras-número; 4) passar o dedo correndo pelos objetos e recitar uma seqüência numérica sem estabelecer correspondência um- 
para-um. Essas violações não poderiam acontecer se o conhecimento fosse inato.

Diante destes resultados, Fuson e colaboradores (Fuson e cols., 1982, 1983, 1985) sugerem que o ato de contar é inicialmente um ato destituído de conhecimento numérico per se, como tinha afirmado Piaget (Piaget \& Szeminska, 1975) anteriormente. De acordo com a hipótese de Fuson e seus colaboradores, a contagem oral é no começo uma recitação de palavras imitando um comportamento aprendido sócio-culturalmente como se fosse um repetir de uma canção de roda que as crianças aprendem nessa idade. Com o aumento da participação das crianças em atividades lúdicas e cotidianas, nas quais elas são estimuladas a usar a contagem oral para contar vários e diversos agrupamentos com vários objetivos, os procedimentos de contar tornam-se mais exercitados, diminuindo, assim, a ocorrência de erros e guiando a criança para o desenvolvimento dos conceitos numéricos.

Outro estudo que levantou falhas no argumento inatista foi conduzido por Briars e Siegler (1984). Estes autores investigaram se as crianças de três, quatro e cinco anos seriam capazes de diferenciar contagens que violavam os princípios conceituais (inatos), portanto incorretas, das contagens que apesar de não-convencionais (da direita para a esquerda, ou do meio para a esquerda e depois do meio para a direita) eram corretas e, assim, não violavam os princípios conceituais. As crianças assistiram a um boneco executar a contagem e depois tinham que dizer se a contagem do boneco estava correta ou incorreta. Os resultados deste estudo mostraram que as crianças de idades de três, quatro anos e algumas de cinco anos não conseguiram diferenciar a contagem nãoconvencional, mas correta, da contagem incorreta. Elas julgaram as duas como erradas na maioria dos testes, embora todas estas crianças soubessem contar corretamente. Os investigadores, assim, concluíram que as crianças, primeiramente, aprendem os procedimentos de contagem e, posteriormente, constroem os conceitos numéricos. Sendo assim, os procedimentos precedem os princípios conceituais e estes últimos, então, não podem guiar a aquisição da contagem verbal.

Os investigadores que defendiam as hipóteses inatistas para aquisição da contagem oral argumen- taram que as tarefas usadas nos testes destes estudos eram muito difícieis para as crianças, causando, assim, uma performance empobrecida apesar da competência numérica conceitual inata (Gelman \& Greeno, 1989; Gelman \& Meck, 1983; Gelman, Meck \& Merkin, 1986). Em outras palavras, os inatistas argumentaram que as crianças tinham a competência conceitual desde o nascimento, mas não conseguiam obter uma boa performance nos testes de laboratório porque as tarefas usadas eram muito dificeis. Os inatistas defendiam, assim, uma continuidade entre as competências numéricas dos bebês com a das crianças de idade pré-escolar.

Os pesquisadores que argumentavam que a contagem é adquirida durante o processo de desenvolvimento da criança (Baroody, 1987, 1992, 1993; Briars \& Siegler, 1984; Frye, Braisby, Love, Maroudas \& Nichols, 1989; Fuson, 1988; Siegler \& Robinson, 1982) explicavam a passagem das habilidades quantitativas documentadas no bebê para as habilidades das crianças de idade pré-escolar de forma descontínua. Isto é, se há uma habilidade numérica no bebê, esta não permanece a mesma nas idades mais avançadas da infância. A habilidade numérica da criança pré-escolar passa por transformações qualitativas e quantitativas.

\section{Estão os conceitos e os procedimentos relacio- nados? Ou separados?}

Se por um lado todos estes estudos evidenciaram que o ato de contar requer várias habilidades de ordens cognitiva, motora e lingüística, por outro, estes mesmos estudos instituíram relações dicotômicas entre conceitos e procedimentos, como também, entre competência e performance e entre adquirido e inato. Desta forma restabeleceu-se o tradicional debate na área do desenvolvimento numérico em crianças da educação infantil (Baroody, 1992; Siegler, 1991), o qual foi discutir se a contagem oral é guiada por conceitos inatos, ou se a contagem é adquirida durante o processo de desenvolvimento através da prática sócio-cultural de usar procedimentos de contar.

Foram vários os estudos conduzidos por pesquisadores de diversas orientações teóricas para buscar uma solução para este polarizado debate. 
A pesquisadora Wynn (1990, 1992a), por exemplo, usou duas tarefas simples para coletar dados sobre o conceito de cardinalidade dos numerais presentes na contagem oral das crianças de dois anos e meio a cinco anos de idade. A primeira tarefa foi "Me dê N" no qual as crianças eram apresentadas com uma cesta de dinossauros e então eram solicitadas pelo pesquisador para dar um determinado número ("Me dê dois dinossauros"). A segunda tarefa consistia em a criança identificar, entre duas cartas contendo fotos de conjuntos de objetos homogêneos, qual a carta que correspondia a uma determinada quantidade; por exemplo: "Você pode me mostrar três flores?". Ambas as tarefas não apresentavam grande grau de dificuldade, eliminando, assim, a possibilidade de problemas na performance durante os testes. Wynn (1990) também pediu que as crianças contassem um conjunto com oito objetos. Os resultados daqueles dois estudos de Wynn (1990, 1992a) (um transversal e outro longitudinal) mostraram que, apesar de todas as crianças serem capazes de contar pelo menos até seis corretamente, elas não sabem o exato valor cardinal de todos os números na sua lista de contagem oral. Por exemplo, quando solicitadas a produzir e identificar uma quantidade, algumas crianças (geralmente as mais novas) só conseguiam produzir e identificar a quantidade "um" com exatidão, outras (um pouco mais velhas) somente "um e dois", outras somente "um, dois e três". Também foi demonstrado que é de mais ou menos seis meses o período entre a aquisição do significado cardinal de um numeral para outro; isto é, a criança primeiro constrói o conceito de "um", depois de "dois" e depois de "três" de forma gradual. Adicionalmente, a autora mostrou que a maioria das crianças não usa espontaneamente a contagem oral para solucionar problemas e, também, comete erros de contagem que violam princípios de correspondência um-para-um.

Os estudos de Wynn (1990, 1992a), portanto, sugerem que inicialmente a contagem oral é, como havia sugerido anteriormente Fuson (1988), um procedimento aprendido socialmente e, inicialmente, desprovido de significado numérico per se.

Mas Wynn (1992a, 1996), todavia, argumenta que para um conceito se desenvolver é necessário que o mesmo seja precedido e sustentado por mecanismos cognitivos de representações mentais, os quais, a autora afirma, são de natureza inata. A hipótese de Wynn é de que as palavras "um" "dois" "três" "quatro", por exemplo, são rótulos que ao ressaltarem o aspecto numérico precisam ser casados com as representações mentais de quantidades que o bebê humano constrói a partir de seu inato senso de número de uma forma contínua.

Com o intuito de mostrar que há uma mudança qualitativa da representação mental do bebê (seja lá qual for o mecanismo cognitivo que a sustente) para as habilidades numéricas da criança de idade préescolar (entre dois e seis anos) e, ainda, que é improdutiva a separação entre conceitos e procedimentos (ou entre competência e performance), recentes estudos demonstraram que existe uma interconectividade entre conceitos e procedimentos (Baroody \& Wilkins, 1999; Baroody, 2003; Le Corre, Van de Walle, Brannon, \& Carey, 2006; Mix e cols, 2002a; Rittle-Johnson \& Siegler, 1998). Por exemplo, quando crianças estão contando objetos elas, geralmente, apontam para cada objeto e recitam um numeral concomitantemente "um, dois, três, cinco, sete." Este comportamento requer, ao mesmo tempo, conhecimentos de procedimentos e conceitos que podem mútua e dinamicamente se influenciarem. Baroody (2003) afirmou que sem os procedimentos, as crianças não teriam o retorno de sua ação para, assim, criar, modificar, e expandir seus conceitos. E sem o conhecimento conceitual as crianças não poderiam saber como e porquê usar determinados procedimentos

Um outro exemplo deste intuito de mostrar a interconexão entre conceitos e procedimentos é encontrado nas pesquisas de Mix (1999a, 1999b) sobre a relação entre a capacidade de julgar equivalência numérica e a contagem verbal. A autora estudou o desenvolvimento da capacidade de julgar equivalência em crianças entre dois e quatro anos e meio de idade. Manipulou diferentes materiais e condições de apresentação dos conjuntos com o objetivo de verificar se tais condições influenciavam diferentemente o desempenho das crianças. Os resultados destes estudos mostraram que a característica dos materiais e o conhecimento de contagem 
influenciam a habilidade de a criança perceber equivalência e que o desenvolvimento desta habilidade é gradual. Assim, as crianças menores de dois anos e meio, que ainda não sabem contar, somente são capazes de reconhecer como equivalentes os conjuntos homogêneos de pouco itens apresentados simultaneamente; portanto, as mesmas usam as informações de ordem perceptiva para fazer seus julgamentos de equivalência. As crianças maiores de três a três anos e meio, que têm um conhecimento maior da contagem, percebem como equivalentes conjuntos constituídos de materiais completamente heterogêneos, mas apresentados simultaneamente. Somente as crianças com quatro anos e mais, que tinham um nível proficiente de contagem, conseguiram identificar equivalência em todas as situações. Essas crianças conseguiram abstrair que o fator comum no julgamento de equivalência numérica é o número dos itens dos conjuntos, independente de suas aparências. Assim, ficou demonstrada a dinâmica interconexão entre procedimentos e conceitos no desenvolvimento da capacidade de abstrair equivalências numéricas. Esses resultados foram os mesmos encontrados no estudo de Barbosa (2004).

O que estes estudos revistos mostraram é que, não é necessário postular a existência de habilidades numéricas inatas para explicar o desenvolvimento dos conceitos numéricos e do ato de contar. $\mathrm{O}$ ato de contar, por exemplo, é um instrumento cultural que as crianças começam a adquirir por volta de dois anos de idade na sua interação com o mundo físico, social, cultural e histórico. Mas a contagem não é uma simples internalização e posterior repetição de comportamentos aprendidos com os outros. Mais do que isso, o ato de contar se constrói, talvez concomitantemente, com a internalização de conceitos e procedimentos que são socialmente e culturalmente mediados e estão dinamicamente inter-relacionados. Este processo dinâmico e ativo de internalização pode envolver processos perceptivos e cognitivos de domínio gerais, entre os quais está o processo de visualização chamado subitizing, os processos de individuação dos objetos, e a aquisição dos nomes dos numerais. Talvez, concordando com a tese de Mix e Sandhofer (2007), não seja necessário hipotetizar sobre um senso de número inato, pois as crianças podem chegar ao mesmo desenvolvimento usando processos cognitivos gerais.

\section{O sentido de número é mais que um, dois, três}

É possível perceber nesta breve revisão dos estudos sobre o desenvolvimento do sentido de número em crianças pequenas que o seu desenvolvimento é progressivo mas não é linear. Como vimos anteriormente, os bebês são capazes de identificar diferentes quantidades, mas a criança de dois anos parece não saber o que é "dois" e "três". Mesmo quando a criança sabe que as palavras "dois" e "três" estão relacionadas a numerais, leva um tempo para ela entender o valor cardinal desta quantidade e, mais um tempo, para usar a seqüência numérica para responder perguntas sobre a quantidade de um conjunto de elementos. Mais importante, ainda, é perceber que a criança real, presente nas salas de educação infantil, não é a criança dos laboratórios de pesquisa, que em idade $\underline{x}$ faz $\underline{w}$ e em idade $\underline{y}$ faz $\underline{m}$. As crianças exibem uma multiplicidade de comportamentos, estratégias e capacidades que podem ser expressas simultaneamente ou separadas e que, também, podem ser mais sofisticadas ou menos sofisticadas de acordo com o contexto (Mix, 2002).

É importante perceber que o ato de contar, assim como qualquer outra habilidade cognitiva, não é um fenômeno do tipo tudo-ou-nada; ou seja, se a criança sabe contar objetos, então, ela sabe contar tudo e qualquer outra coisa. Muito pelo contrário, o processo de desenvolvimento do ato de contar é um processo gradual. Uma criança pode contar corretamente cinco objetos arrumados horizontalmente, e errar a contagem de cinco objetos arrumados em círculo. Isso não quer dizer que a criança não saiba contar. Isso quer dizer que o arranjo espacial dos objetos influencia o ato de contar. É mais fácil contar objetos arrumados horizontalmente do que objetos arrumados em círculo (Fuson, 1988). Como também, é mais fácil contar objetos do que contar ações, que não possuem uma representação física (Wynn, 1990, 1992a). Assim, a natureza da tarefa e os materiais usados como suporte influenciam a performance da criança e mostram variações de seu desempenho.

Pesquisadores têm argumentado que, apesar desta variação ser intrínseca ao processo de 
desenvolvimento humano, a mesma é mais visível na criança devido à natureza de sua aprendizagem, a qual é marcada pelo contexto (Nelson, 1996; Mix, 2002; Thelen \& Smith, 1994). Assim, é possível que a criança exiba um comportamento mais competente em um contexto, e menos competente em outro contexto, dependendo dos instrumentos de suporte a que a criança tem acesso para mediar sua aprendizagem (Vygotsky, 1978). Estes instrumentos de suporte que sustentam a aprendizagem, e que constituem o contexto desta mesma aprendizagem, são a linguagem utilizada, os materiais usados, a organização do espaço físico, a qualidade das interações sociais propiciadas por este espaço dentro do contexto lingüístico-cultural, as possibilidades de autonomia de pensamento e de ação; enfim, todo o aparato envolvido no ato educativo. Durante o processo de desenvolvimento da criança, sua aprendizagem passa a ser menos dependente do contexto onde esta se realiza (Nelson, 1996). O desenvolvimento cognitivo, então, passa a ser entendido como processo de criação de ligações entre estes contextos de aprendizagem inicialmente separados (Barbosa, 2004; Baroody, 2003).

A construção do sentido de número, portanto, requer mais do que o conhecimento cardinal de "um", "dois" e "três". Podemos pensar o desenvolvimento do sentido de número como criação de conexões e relações flexíveis entre idéias e habilidades de caráter numérico-cognitivas que podem, inicialmente, estar separadas e em um determinado contexto (Lefevre e cols, 2006). As condições materiais, sociais e psicológicas que sustentam este processo de construção de conexões podem influenciar a qualidade destas conexões, as quais, por sua vez, podem influenciar a qualidade do sentido de número.

Assim, a qualidade e a profundidade do sentido de número desenvolvido por uma criança pode ser um indicador de como esta criança está entendendo e apreendendo conhecimentos numérico-matemáticos.

Aliás, este é o argumento apresentado por Spinillo (2006) quando discute a importância do sentido de número na educação matemática. A autora enumera e investiga alguns comportamentos e habilidades que podem ser indicadores de de- senvolvimento de um bom sentido de número, tais como: (1) computação numérica flexível, (2) julgamentos quantitativos e inferência, (3) uso de âncora (conhecimentos anteriores servem de referência), (4) reconhecer resultados adequados ou absurdos, (5) reconhecer magnitude relativa e absoluta, (6) compreender como as operações aritméticas atuam sobre os números, (7) escolher e usar instrumentos de representação adequados e (8) reconhecer usos, significados e funções dos números no cotidiano.

Finalizando, podemos dizer que os estudos produzidos até agora apontam para uma dinâmica interconexão entre conceitos e procedimentos durante o desenvolvimento do sentido de número na infância. A construção de conceitos e habilidades numéricas é um processo gradual, variável, individual, e intrinsecamente atrelado ao contexto onde ocorre. A organização de contextos de aprendizagem que levem em consideração a experiência individual da criança, a expressão de suas idéias, a experimentação de suas hipóteses e a troca de informações entre pares é de grande importância para oportunizar a emergência de indicadores comportamentais de um bom desenvolvimento do sentido de número na educação da criança pequena.

\section{Referências}

Antell, S., \& Keating, D. P. (1983). Perception of numerical invariance in neonates. Child Development, 54, 695-701.

Barbosa, H. (2004). Numerical abilities of preschool children with atypical development: A developmental description. Unpublished doctoral thesis, Boston University, Boston, MA.

Baroody, A. J. (1987). Children's mathematical thinking: A developmental framework for preschool, primary, and special education teachers. New York, NY: Teachers College Press.

Baroody, A. J. (1992). The development of preschoolers' counting skills and principles. In J. Bideaud, C. Meljac \& J. P. Fisher (Eds.). Pathways to number: Children's developing numerical abilities (pp. 99-26). Hillsdale, NJ: Lawrence Erlbaum Associates. 
Baroody, A. J. (1993). The relationship between the order-irrelevance principle and counting skill. Journal for Research in Mathematics Education, 24, 415-427.

Baroody, A. J. (2003). The development of adaptive expertise and flexibility: The integration of conceptual and procedural knowledge. In A. J. Baroody \& A. Dowker, A. (Eds.). The development of arithmetic concepts and skills: Constructing adaptive expertise (pp. 1-33). Hillsdale, NJ: Lawrence Erlbaum Associates.

Baroody, A. J., \& Wilkins, J. L. M. (1999). The development of informal counting, number, and arithmetic skills and concepts. In J. V. Copley (Ed.). Mathematics in the early years (pp. 4865). Washington, DC: National Association for the Education of Young Children.

Briars, D., \& Siegler, R. S. (1984). A featural analysis of preschoolers' counting knowledge. Developmental Psychology, 20, 607-618.

Butterworth, B. (1999). What counts: How every brain is hardwired for math. New York, NY: The Free Press.

Carey, S. (1991). Knowledge acquisition: Enrichment or conceptual change? In S. Carey \& R. Gelman (Eds). The epigenesis of mind: Essays on biology and cognition (pp. 257-291). Hillsdale, NJ: Lawrence Erlbaum Associates.

Carey, S. (2001). Whorf versus continuity theorists: Bringing data to bear on the debate. In $\mathrm{M}$. Bowerman \& C. Levinson (Eds.). Language acquisition and conceptual development (pp. 185-214). Cambridge, MA: Cambridge University Press

Carey, S., \& Spelke, E. (1994). Domain-specific knowledge and conceptual change. Mapping the mind. In L. A. Hirschfield \& S. Gelman (Eds). Domain specificity in cognition and culture (pp. 169-200). New York, NY: Cambridge University Press.

Chi, M. T., \& Klahr, D. (1975). Span and rate of apprehension in children and adults. Journal of Experimental Child Psychology, 19, 434-439.
Clearfield, M. W., \& Mix, K. S. (1999). Number versus contour length in infants' discrimination of small visual sets. Psychological Science, 10, 408-411.

Clearfield, M.W., \& Mix, K. S. (2001). Amount versus number: Infants' use of area and contour length to discriminate small sets. Journal of Cognition and Development, 2, 243-260.

Cooper, R. G., Jr. (1984). Early number development: Discovering number space with addition and subtraction. In C. Sophian (Ed.). Origins of cognitive skills: The Eighteenth Annual Symposium on Cognition (pp. 157-192). Hillsdale, NJ: Lawrence Erlbaum Associates.

Dehaene, S. (1997). The number sense: How the mind creates mathematics. New York, NY: Oxford University Press.

Feigenson, L., Carey, S., \& Spelke, E. S. (2002). Infants' discrimination of number vs. continuous extent. Cognitive Psychology, 44, 33-66.

Frye, D., Braisby, N., Love, J., Maroudas, C., \& Nicholls, J. (1989). Young children's understanding of counting and cardinality. Child Development, 60, 1158-1171.

Fuson, K. (1988). Children's counting and concepts of number. New York, NY: Springer-Verlag.

Fuson, K., Richards, J., \& Briars, D. (1982). The acquisition and elaboration of the number word sequence. In C. J. Bainerd (Ed.). Children's logical and mathematical cognition (pp. 33-92). New York, NY: Springer-Verlag.

Fuson, K. C., Secada, W. G., \& Hall, J. W. (1983). Matching, counting, and conservation of numerical equivalence. Child Development, 54, 91-97.

Fuson, K. C., Pergament, G. G., Lyons, B. G., \& Hall, J. W. (1985). Children's conformity to the cardinality rule as a function of set size and counting accuracy. Child Development, 56, 1429-1436.

Gelman, R. (1991). Epigenetic foundations of knowledge structures: Initial and transcendent constructions. In S. Carey \& R. Gelman (Eds.). The epigenesis of mind: Essays on biology and cognition (pp. 293-322). Hillsdale, NJ: Lawrence Erlbaum Associates. 
Gelman, R., \& Gallistel, C. R.(1978). The child's understanding of number. Cambridge, MA: Harvard University Press.

Gelman, R., \& Greeno, J. G. (1989). On the nature of competence: principles for understanding in a domain. In L. B. Resnick (Ed.). Knowing and learning: Issues for a cognitive science of instruction (pp. 125-186). Hilsdale, NJ: Lawrence Erlbaum Associates.

Gelman, R., \& Meck, E. (1983). Preschoolers' counting: Principles before skill. Cognition, 13, 343-359.

Gelman, R., Meck, E., \& Merkin, S. (1986). Young children's numerical competence. Cognitive Development, 1, 1-29.

Huttenlocher, J., Duffy, S., \& Levine, S. (2002). Infants and toddlers discriminate amount: Are they measuring? Psychological Science, 13, 244-249.

Karmiloff-Smith, A. (1992). Beyond modularity: A developmental perspective in cognitive science. Cambridge, MA: MIT Press/Bradford Books.

Le Corre, M., Van de Walle, G., Brannon, E. M., \& Carey, S. (2006). Re-visiting the performance/ competence debate in the acquisition of counting as a representation of the positive integers. Cognitive Psychology, 52, 130-169.

Lefevre, J. A., Smith-Chant, B. L., Fast, L., Skwarchuk, S. L., Sargla, E., Arnup, J. S., PennerWilger, M., Bisanz, J., \& Kamawar, D. (2006). What counts as knowing? The development of conceptual and procedural knowledge of counting from kindergarten through grade 2. Journal of Experimental Child Psychology, 93, 285-303.

Mix, K. S. (1999a). Similarity and numerical equivalence: Appearances count. Cognitive Development, 14, 269-297.

Mix, K. S. (1999b). Preschooler's recognition of numerical equivalence: Sequential sets. Journal of Experimental Child Psychology, 74, 309-332.

Mix, K. S. (2002). The construction of number concepts. Cognitive Development, 17, 1345-1363.
Mix, K. S., Huttenlocher, J., \& Levine, S. C. (2002a). Quantitative development in infancy and early childhood. New York, NY: Oxford University Press.

Mix, K. S., Huttenlocher, J. \& Levine, S. C. (2002b). Multiple cues for quantification in infancy: Is number one of them? Psychological Bulletin, 128, 278-294.

Mix, K. S., Levine, S. C., \& Huttenlocher, J. (1997). Numerical abstraction in infants: Another look. Developmental Psychology, 33, 423-428.

Mix, K., Sandhofer, C., \& Baroody, A. (2005). Number words and number concepts: The interplay of verbal e non-verbal quantification in early chilhood. In R. V. Kail (Series Ed.). Advances in child development and behavior: Vol. 33 (pp. 305-346). New York, NY: Academic Press.

Mix, K., \& Sandhofer, C. (2007). Do we need a number sense? In M. J. Roberts (Ed.). Integrating the mind (pp.293-326). Hove, UK: Psychology Press.

Nunes, T. \& Bryant, P. (1997). Crianças fazendo matemática. Porto Alegre: Artes Médicas.

Nelson, K. (1996). Language in cognitive development: Emergence of the mediated mind. New York, NY: Cambridge University Press.

Piaget, J., \& Szeminska, A. (1975). A gênese do número na criança (C. M. Oiticica, trad). Rio de Janeiro, RJ: Zahar.

Rittle-Johnson, B., \& Siegler, R. S. (1998). The relation between conceptual and procedural knowledge in learning mathematics: A review of the literature. In C. Donlan (Ed.). The development of mathematical skills (pp. 75-110). Hove, UK: Psychology Press.

Siegler, R. (1991). In young children's counting, procedures precede principles. Educational Psychology Review, 3, 127-135. 
Siegler, R. S., \& Robinson, M. (1982). The development of numerical understanding. In H.W. Reese \& L.P. Lipsitt (Series Eds.). Advances in child development and behavior: Vol. 16 (pp. 241-313). New York, NY: Academic Press.

Spelke, E., Breinlinger, K., Macomber, J., \& Jacobson, K. (1992). Origins of knowledge. Psychological Review, 99, 605-632.

Spelke, E., \& Dehaene, S. (1999). Biological foundations of numerical thinking: Response to T. J. Simon. Trends in Cognitive Sciences, 3, 365-366.

Spelke, E., \& Tsivkin, S. (2001). Initial knowledge and conceptual change: Space and number. In M. Bowerman \& C. Levinson (Eds.). Language acquisition and conceptual development (pp. 70-97). Cambridge, UK: Cambridge University Press.

Spinillo, A.G. (2006). O sentido de número e sua importância na educação matemática. In M. R. Brito (Org.). Soluções de problemas e a matemática escolar (pp. 83-111). Campinas, SP, Alínea.

Starkey, P., \& Cooper, R.G., Jr. (1980). Perception of number by human infant. Science, 210, 1033-1035.

Starkey, P., \& Spelke, E. (1983). Detection of intermodal numerical correspondences by human infants. Science, 222, 179-181.

Strauss, M. S., \& Curtis, L. E. (1981). Infant perception of numerosity. Child Development, 52, 1146-1152.

Strauss, M. S., \& Curtis, L. E. (1984). Development of numerical concepts in infancy. In C. Sophian (Ed.). Origins of cognitive skills: The Eighteenth Annual Symposium on Cognition (pp. 131-155). Hillsdale, NJ, Lawrence Erlbaum Associates.

Thelen, E., \& Smith, L. B. (1994). A dynamic systems approach to the development of cognition and action. Cambridge, MA: The MIT Press.

Vygotsky, L. S. (1978). Mind and society. Cambridge, MA: Harvard University Press.
Wakeley, A., Rivera, S., \& Langer, J. (2000). Can young infants add and subtract? Child Development, 71, 1525-1534.

Wynn, K. (1990). Children's understanding of counting. Cognition, 36, 155-193.

Wynn, K. (1992a). Children's acquisition of the number words and the counting system. Cognitive Psychology, 24, 220-251.

Wynn, K. (1992b). Addition and subtraction by human infants. Nature, 358, 749-750.

Wynn, K. (1992c). Evidence against empiricist accounts of the origins of numerical knowledge. Mind and Language, 7, 315-332.

Wynn, K. (1996). Infant's individuation and enumeration of physical actions. Psychological Science, 7, 164-169.

Wynn, K. (1998a). Psychological foundations of number: numerical competence in human infants. Trends in Cognitive Science, 2, 296-303.

Wynn, K. (1998b). Numerical competence in infants. In C. Donlan (Ed.). The development of mathematical skills. Studies in developmental psychology (pp. 3-25). Hove, UK: Psychology Press/Taylor \& Francis.

A elaboração deste artigo foi fruto dos estudos da autora que contou com bolsa de doutorado pleno no exterior pela CAPES.

Artigo recebido em 10/09/2007.

Aceito para publicação em 17/08/2007.

Endereço para correspondência:

Rua das Araras, 432. Lagoa da Conceição, CEP 88062-075. Florianopolis-SC, Brasil. E-mail: heloiza@hbarbosa.org

Heloiza Helena de Jesus Barbosa é Pedagoga; Doutora em Educação pela Boston University 
194 Paidéia, 2007, 17(37), 181-194

com Pós-Doutorado concluído na University of California at Berkeley. Atualmente conclui o seu segundo Pós-Doutorado na Universidade Federal de Santa Catarina, Centro de Ciências da Educação, com auxílio do CNPq. 